Research Article

\title{
Recommendation with Diversity: Mass Diffusion Model Based on Trust Network and Object Reputation
}

\author{
Yun Bai (iD) and Wandong Cai \\ School of Computer Science, Northwestern Polytechnical University, Xi'an 710072, China \\ Correspondence should be addressed to Yun Bai; 395943619@qq.com
}

Received 24 February 2021; Revised 25 June 2021; Accepted 31 July 2021; Published 10 August 2021

Academic Editor: Juan C. Cano

Copyright () 2021 Yun Bai and Wandong Cai. This is an open access article distributed under the Creative Commons Attribution License, which permits unrestricted use, distribution, and reproduction in any medium, provided the original work is properly cited.

\begin{abstract}
The traditional mass diffusion recommendation algorithm only relies on the user's object collection relationship, resulting in poor recommendation performance for users with small purchases (i.e., small-degree user), and it is difficult to balance the accuracy and diversity of the recommendation system. This paper introduces the trust relationship into the resource allocation process of the traditional mass diffusion algorithm and proposes the Dual Wing Mass Diffusion model (DWMD), which constructs a dual wing graph based on trust relationships and object collection relationships. Implicit trust is mined according to the network structure of the trust relationship and integrated into the resource allocation process, and then merging the positive effects of object reputation on a recommendation through tunable scaling parameters. The user controls the tunable scaling parameter to achieve the best recommendation performance. The experimental results show that the DWMD method significantly improves diversity and novelty while ensuring high accuracy and effectively improves the accuracy and diversity balance. The improved recommendation performance for small-degree users proves that the trust relationship can effectively alleviate the generalized cold start problem of the recommendation algorithm for users who collect a small number of objects.
\end{abstract}

\section{Introduction}

The explosive growth of Internet information and the prosperity of e-commerce has made the problem of information overload increasingly serious. The mass of commodities not only makes it difficult for consumers to choose their favorite commodities but also makes it difficult for commodity producers to distinguish their single products from the mass of commodities. It has been proved that the recommendation system is an effective information filtering method, which can help users quickly obtain target information and solve the problem of information overload [1]. At present, most of the research on recommendation algorithms focuses on the accuracy of recommendations while ignoring the diversity and novelty of recommendation results. This leads to the user's choice being limited to a relatively "narrow" set of objects by the recommendation system, and the user's potential interest may be ignored and low-profile new products are silent because they have not been recommended for a long time, such as the current prominent popular recommendation issues in e-commerce websites. Users are recommended to buy objects that are popular and unsuitable for them, but the objects that users really need cannot be effectively recommended due to the low exposure, which is not conducive to converting users' potential needs into actual needs and increasing sales. Research on the diversity and novelty of recommendation systems has a positive effect on improving user satisfaction and product sales. Although the method of increasing diversity and novelty by reducing accuracy is easy to implement, it does not achieve the best recommendation effect. The best recommendation effect is that the recommendation result has both high accuracy and high diversity and novelty. Therefore, the current challenge we are facing is to increase diversity and novelty while ensuring accuracy.

In recent years, many scholars have conducted research on improving the performance of recommendation systems. Research [2-6] mostly improves recommendation 
performance based on user ratings and social relationships. Tahmasebi et al. [2] propose a hybrid recommendation method based on profile expansion technique to improve the accuracy and coverage of the recommendation system. Ahmadian et al. [4] study the positive impact of social information among the users and the temporal information of ratings on the accuracy and coverage of the recommendation system. Ahmadian et al. [6] proposed a probabilistic method to predict reliable ratings for the unseen items, which alleviates the cold start and data sparsity problems. There are also some studies that apply physical diffusion and physical heat conduction theories to recommendation systems based on network structures, which opens up new directions for the research of recommendation algorithms. The recommendation algorithm based on physical dynamics has received widespread attention because of its strong adaptability, high recommendation accuracy, and low environmental requirements. The algorithm includes Mass Diffusion (MD) [7], Heat Conduction algorithm (HC) [8], and various methods derived on this basis, such as Hybrid ProbS and HeatS algorithm $(\mathrm{HPH})$ that combines $\mathrm{MD}$ and HC [9], Biased Heat Conduction (BHC) [10], Balanced Diffusion recommendation algorithm (BD) [11], Preferential Diffusion (PD) [12], and some other methods [12-15]. These methods restrict the recommendation results to a relatively "narrow" collection of objects due to excessive reliance on the user's choice of objects, and the diversity and novelty of the recommendation are not good.

Social media has developed rapidly, and there is a wealth of user related information in social networks. Studies have shown that the trust relationship between users affects users' decision-making [16-19]. These rich user relationship data provide a new idea for improving the accuracy, diversity, and novelty of the recommendation system. Some researchers combine trust relationships with Mass Diffusion algorithms for research. The mass diffusion based on trust relationship [20], Hybrid Trust $\mathrm{MD}+\mathrm{HC}$ method [21], CosRA + T method [22], etc. are all using the trust between users as the weight for resource allocation. Although these studies have improved the recommended performance to a certain extent, they still have the following shortcomings: (1) The calculation of the trust between users still relies on the object selection relationship, and the additional trust information is not fully utilized to construct the trust measurement method, which leads to the recommendation results tending to popular items, and the diversity and novelty performance are not improved well. (2) These studies did not make full use of user rating information, which exacerbated the sparseness and singularity of data to a certain extent, and did not consider the impact of user reputation on recommendations. (3) These studies have a good recommendation effect for users with large purchases (i.e., large-degree users) or sufficient trust information, while the recommendation performance for small-degree users with fewer purchases or trust information has not been carried out. Small users are a relatively large group that cannot be ignored in the real recommendation system.

In view of the above problems, this paper proposes a dual wing mass diffusion model based on trust network and object reputation (DWMD for short). The model constructs tripartite graphs according to the trust relationship and object selection relationship and combines the two relations with tunable parameters to balance the dilemma of accuracy and diversity of recommendation. In the network structure based on a trust relationship, the implicit trust relationship is discovered according to the structure of the trust network, and the trust measurement model is constructed. In the network structure based on object selection relationships, rating information is fully utilized to improve the accuracy of recommended items. The two network structures are combined by tunable parameters.

The proposed method provides the following contributions:

(1) Constructed a user trust measurement model based on a trust relationship and proposed a resource allocation plan based on trust

(2) Constructed an evaluation-based mass diffusion model to allocate resources based on user rating

(3) The trust relationship and the object selection relationship are combined to construct a tripartite graph

The remainder of this paper is organized as follows. Section 2 introduces the proposed DWMD method. Section 3 introduces the used datasets and performance evaluation metrics. Section 4 presents our main results. Finally, Section 5 provides concluding remarks.

\section{Dual Wing Mass Diffusion Model Based on Trust Network and Object Reputation}

The recommendation system can be described by a "userobject" binary network $G(U, O, E)$, where $U=\left\{u_{1}, \ldots, u_{m}\right\}$ is the set of users, $O=\left\{O_{1}, \ldots, O_{n}\right\}$ is the set of objects, and $E$ is the set of links between users and objects. The bipartite network can be represented by an $m \times n$ adjacency matrix $A$, where the element $a_{i \alpha}=1$ if $o_{\alpha}$ is collected by $u_{i}$, and the element $a_{i \alpha}=0$ if otherwise. The main purpose of the recommendation system is to provide a list of $L$ uncollected objects most likely to need in the recommendation list $O^{L}$ for the target user $u_{i}$. We use $i, j$ for the subscripts of users and $\alpha, \beta$ for objects. The degree of user $u_{i}$, denoted by $k_{i}$, is the number of objects collected by user $u_{i}$. Similarly, the degree of objects $o_{\alpha}$, denoted by $k_{\alpha}$, is the number of users who have collected object $o_{\alpha}$. The trust relationship can be modeled through the user-user bipartite network $G(U, T)$ and represented by an $m \times m$ adjacency matrix $B$, where $b_{i j}=1$ if user $u_{i}$ trusts $u_{j}$, otherwise $b_{i j}=0$. $T$ represents the trust relationship between users.

The Dual wing mass diffusion model is described in three parts. The first part and the second part are the basis of the third part. The first part is to improve recommendation accuracy from the perspective of object reputation, and the second part is to improve recommendation diversity and novelty from the trust relations in social networks. The third part combines object reputation with the trust relationship in social networks to ensure the accuracy of 
recommendations while enhancing the diversity and novelty of recommendations.

2.1. Mass Diffusion with Object Reputation Sensitivity. In the traditional mass diffusion recommendation algorithm, resources are obtained or allocated according to the principle of even distribution, ignoring the impact of object reputation on the recommendation. A rating in the recommendation system represents a user's recognition of an object. Only popular and well-known objects are worth recommending.

This part recommends objects based on their reputation and popularity and proposes a rating-sensitive mass diffusion algorithm that allocates resources. The specific algorithm is shown in Algorithm 1. The specific method is as follows:

Step 1: The initial resources of the objects collected by the target user $u_{i}$ are set to 1 , and the initial resources of the uncollected objects are 0 . That is, the initial resource of an object is as follows:

$$
f_{i \alpha}=a_{i \alpha} .
$$

Step 2: Obtain resources from $o_{\alpha}$ according to $w_{\alpha i}=r_{i \alpha} / R\left(o_{\alpha}\right)$. The resources that user $u_{i}$ obtains from $o_{\alpha}$ is as follows:

$$
f_{i}^{r}=\sum_{\alpha=1}^{n} f_{i \alpha} \times w_{\alpha i}
$$

where $r_{i \alpha}$ is the rating of $o_{\alpha}$ from $u_{i}$, and $R\left(o_{\alpha}\right)$ is the sum of ratings obtained by $o_{\alpha}$.

Step 3: Allocate resources to $o_{\beta}$ according to $w_{i \beta}=r_{i \beta} / R\left(u_{i}\right)$. For an arbitrary object $o_{\beta}$, the final resource received is as follows:

$$
f_{\beta}^{r}=\sum_{i=1}^{m} a_{i \beta} \times f_{i}^{r} \times w_{i \beta}=\sum_{\alpha=1}^{n} \frac{1}{R\left(o_{\alpha}\right)} \sum_{i=1}^{m} \frac{r_{i \alpha} r_{i \beta} a_{i \alpha} a_{i \beta}}{R\left(u_{i}\right)},
$$

where $R\left(u_{i}\right)$ is the sum of ratings made by $u_{i}$. The transfer matrix element $w_{\beta \alpha}^{r}$ is as follows:

$$
w_{\beta \alpha}^{r}=\frac{1}{R\left(o_{\alpha}\right)} \sum_{i=1}^{m} \frac{r_{i \alpha} r_{i \beta} a_{i \alpha} a_{i \beta}}{R\left(u_{i}\right)} .
$$

In Figure 1, users and objects are denoted by circles and squares, respectively. The black solid lines between users and objects represent collection relations and the weight of the lines are the user's ratings to collected objects. The gray circle means the target user. The values next to users and objects are the resources they have after mass diffusion. Comparing Plot (a) to Plot (b), mass diffusion with rating sensitivity is recommended for objects with high ratings but small purchases, while recommendations for objects with large purchases but low ratings are suppressed.

2.2. Mass Diffusion Based on Trust Relationships. In real society, people always tend to choose recommendations made by friends $[23,24]$. Based on this actual situation, this article assumes that target users tend to choose objects purchased or recommended by trusted users. That is, $u_{i}$ trusts $u_{j}$, and $u_{j}$ has collected $o_{\alpha}, o_{\alpha}$ can be recommended to $u_{i}$.

The trust relationships can be modeled through the user-user bipartite network and represented by an $m \times m$ adjacency matrix $B$, where $b_{i j}=1$ if user $u_{i}$ trusts $u_{j}$, otherwise $b_{i j}=0$. The explicit trust relationship is relatively sparse, and the implicit trust relationship hidden in the network has not been used in the recommendation algorithm. This paper digs out implicit trust relationships according to the trust network structure and conducts a mass diffusion model based on both explicit trust and implicit trust to achieve the purpose of improving the recommendation effect. Existing research is to allocate resources based on trust weight that is calculated by the similarity of objects collected by users, which leads to poor recommendation for users collected fewer objects. This paper explores implicit trust from the perspective of the trust network structure and measures the trust values between two users through the network structure, effectively reducing the data sparsity and introducing additional information from the trust network structure to improve the recommendation performance.

\subsubsection{Explicit Trust}

Definition 1. Explicit trust refers to the trust relationship declared by the user, that is, $u_{i}$ expressly trusts $u_{j}$. Trust value between $u_{i}$ and $u_{j}$ is measured by the number of objects jointly selected and the similarity of the ratings. The calculation formula is as follows:

$$
T_{i j}^{\operatorname{sim}}=\frac{\sum_{\alpha=1}^{n} a_{i \alpha} a_{j \alpha}}{\sqrt{K\left(u_{i}\right) K\left(u_{j}\right)}} \operatorname{sim}(i, j),
$$

where $\operatorname{sim}(i, j)$ is the similarity of ratings between $u_{i}$ and $u_{j}$ calculated by the cosine similarity.

2.2.2. Implicit Trust. According to life experience: If two people trust a third person at the same time, there may be a potential trust relationship between them, which is called coupling trust. Similarly, if two people are trusted by a third person at the same time, there may also be a potential trust relationship between them, called cocitation trust. Implicit trust refers to the trust relationship inferred from the structure of the trust network.

(1) Coupling trust: The more users that both $u_{i}$ and $u_{j}$ trusted, the stronger the coupling trust between $u_{i}$ and $u_{j}$. The formula is as follows:

$$
\mathrm{CT}_{i j}=\frac{\sum_{l=1}^{m} b_{i l} b_{j l}}{\operatorname{Out}\left(u_{i}\right)}
$$

where Out $\left(u_{i}\right)$ represents the out-degree of $u_{i}$, that is, the number of users trusted by $u_{i}$. 
Input: "user-object" binary network $G(U, O, E)$, rating matrix $R$, and object user $u_{i}$;

Output: the final resources of objects $\left\{f_{1}^{r}, \ldots, f_{n}^{r}\right\}$;

(1) Initialize object resources according to equation (1);

(2) Calculate the resources that $u_{j}$ obtains from objects (i.e., $f_{j}^{r}, j=1, \ldots, m$ ) according to equation (2);

(3) Calculate the resources of object $o_{\beta}$ (i.e., $f_{\beta}^{r}, \beta=1, \ldots, n$ ) according to equation (3);

Algorithm 1: Algorithm of mass diffusion with object reputation.

Input: "object-user-user" triple network $G(U, O, E, B)$, rating matrix $R$, and object user $u_{i}$;

Output: the final resources of objects $\left\{f_{1}^{\text {trust }}, \ldots, f_{n}^{\text {trust }}\right\}$;

(1) Calculate the explicit trust and implicit trust between $u_{j}$ and $u_{i}$ respectively, according to equations (5) and (8)

(2) Calculate the resources that $u_{j}$ obtains from $u_{i}$ (i.e., $f_{j}^{\text {trust }}$ ) according to equation (9), when $B_{i j}=1$ or $b_{i j}=1(j=1, \ldots, m$ and $j \neq i)$

(3) Calculate the resources of object $o_{\beta}$ (i.e., $f_{\beta}^{\text {trust }}, \beta=1, \ldots, n$ ) according to equation (10)

Algorithm 2: Algorithm of mass diffusion based on trust relationships.

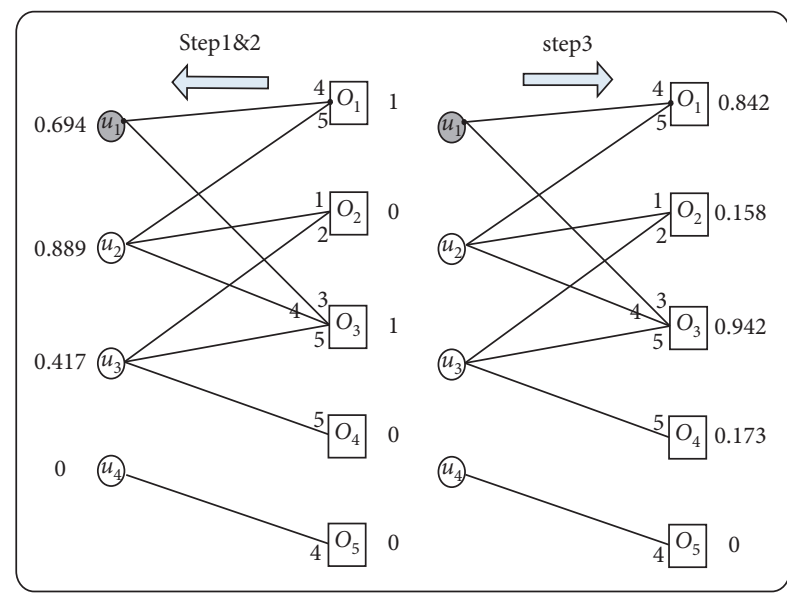

(a)

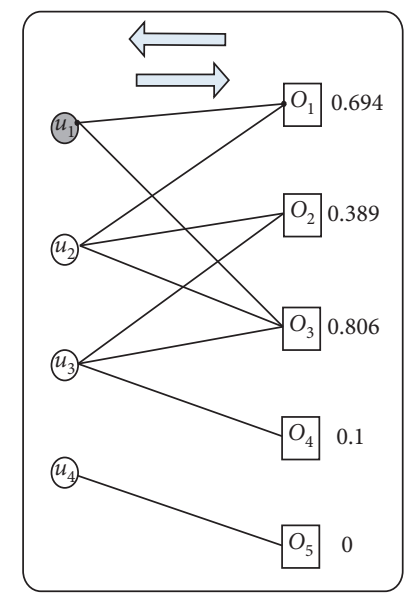

(b)

Figure 1: (a) Mass diffusion with object reputation sensitivity. (b) Traditional mass diffusion without considering object reputation.

(2) Cocitation trust: The more users trust $u_{i}$ and $u_{j}$ together, the stronger the cocitation trust between $u_{i}$ and $u_{j}$. The formula is as follows:

$$
\mathrm{CC}_{i j}=\frac{\sum_{l=1}^{m} b_{l i} b_{l j}}{\operatorname{In}\left(u_{i}\right)}
$$

where $\operatorname{In}\left(u_{i}\right)$ represents the in-degree of $u_{i}$, that is, the number of users who trust $u_{i}$.

(3) Implicit trust measurement:

Definition 2. Implicit trust: there is no explicit trust relationship between $u_{i}$ and $u_{j}$. If $u_{j}$ and the target user $u_{i}$ have both coupling trust and cocitation trust, it is considered that there is implicit trust between $u_{i}$ and $u_{j}$.

The trust weight between $u_{i}$ and $u_{j}$ is as follows:

$$
T_{i j}=\frac{\mathrm{CT}_{i j}+\mathrm{CC}_{i j}}{2}
$$

Construct a user-user-item tripartite graph on the basis of the user-item bipartite graph, as shown in Figure 2, which contains four users $\left\{u_{1}, \ldots, u_{4}\right\}$, the trust relationships, and object collection relationships. The same user corresponds to the dotted line. The explicit trust is represented by a solid black arrow, and the implicit trust is represented by a red dotted arrow. This part of the research will focus on this tripartite diagram for further research.

Users with coupling trust are $\left(u_{1}, u_{2}\right)$ and $\left(u_{1}, u_{4}\right)$. Users with cocitation trust are $\left(u_{1}, u_{3}\right),\left(u_{1}, u_{4}\right),\left(u_{2}, u_{3}\right)$, and $\left(u_{3}, u_{4}\right)$; there is implicit trust between $u_{1}$ and $u_{4}$, which is indicated by the red dashed line in Figure 2. The specific algorithm of mass diffusion based on trust relationships is shown in Algorithm 2 and the specific steps are as follows: 


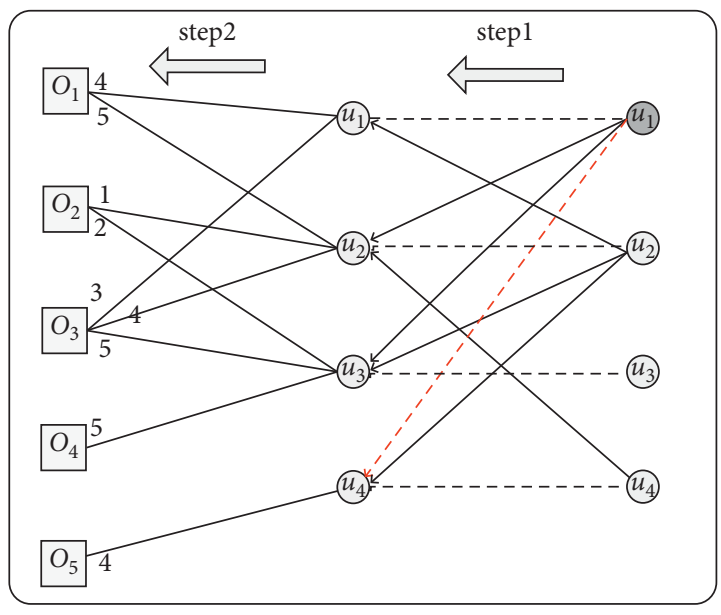

Figure 2: Mass diffusion based on trust relationships. implicit trust users according to weight $T_{i j}$ through the trust network.

$$
f_{j}^{\text {Trust }}=f_{i} B_{i j} T_{i j}+f_{i} b_{i j} T_{i j}^{\text {sim }},
$$

where $f_{i}$ is the resources of $u_{i}$ obtains from collected objects, $b_{i j}$ represents the explicit trust between $u_{i}$ and $u_{j}$. The implicit trust is denoted by $B_{i j}=\left\{\begin{array}{ll}1, & T_{i j} \neq 0, \\ 0, & T_{i j}=0\end{array}\right.$. $B_{i j}=1$ means that $u_{i}$ and $u_{j}$ have the implicit trust; otherwise, there is no implicit trust between them.

Step 2: User $u_{j}$, who is trusted by the target user $u_{i}$, allocates resources to the collected objects according to the weight $w_{j \beta}=r_{j \beta} / R\left(u_{j}\right)$; then the resources that an arbitrary object $o_{\beta}$ obtains from the trust network are as follows:

Step 1: Target user $u_{i}$ allocates initial resources to explicit trust users according to weights $T_{i j}^{\mathrm{sim}}$ and to

$$
f_{\beta}^{\text {Trust }}=\sum_{j=1}^{m} f_{j}^{\text {Trust }} \times w_{j \beta} \times f_{j \beta}=\frac{1}{\sqrt{\operatorname{Out}\left(u_{i}\right) \operatorname{In}\left(u_{i}\right)}} \sum_{j=1}^{m} \frac{b_{i j} r_{j \beta} f_{j \beta}}{R\left(u_{j}\right)} \sqrt{\sum_{l=1}^{m} b_{i l} b_{j l} b_{l i} b_{l j} .}
$$

2.3. Building Dual Wing Model Mass Diffusion Model. In the recommendation system, considering the ratings and trust relationships comprehensively, the resources obtained by the object $o_{\beta}$ are divided into two parts: one is obtained based on object collection relationships which considered the effecting of object reputation, and the other is obtained based on the trust network. Therefore, a double-wing model combining object reputation affection and trust relationships by a tunable parameter is proposed, as shown in Figure 3. The dashed box on the right is the resource obtained from the object collection relationships, and the dashed box on the left is the resource obtained from the trust relationships. The final resource obtained by an arbitrary object $o_{\beta}$ is as follows:

$$
f_{\beta}=(1-\lambda) f_{\beta}^{r}+\lambda f_{\beta}^{\text {Trust }} .
$$

\section{Data and Metrics}

3.1. Datasets. To measure the effect of the algorithm, this paper uses the Epinions and Cao data set [19] for the experiments. Both datasets contain trust relationships and ratings for objects. The rating mechanism of $1 \sim 5$ points is adopted to represent the degree of preference of collected objects in ascending order. The detailed information of the two data sets is shown in Table 1.

This paper has conducted 10 cross-validations and takes an average of 10 experiments as the final result. In each cross-validation, $90 \%$ of the data is used as the training set, with $10 \%$ being the test set.

3.2. Evaluation Metrics. The accuracy, diversity, and novelty of the recommendation system are important indicators for evaluating the performance of the recommendation. This article introduces Precision, Recall, and Ranking Score as accuracy metrics. The diversity metrics are measured by Hamming distance and intrasimilarity. The novelty metrics is measured by Novelty. Ranking Scores are independent of the recommendation list's length $L$, while the rest metrics are all $L$-dependent. Larger Precision and Recall and lower Ranking Score suggest higher accuracy. Larger Hamming distance and smaller intrasimilarity mean better diversity. The calculation formula of each metric is as follows.

3.2.1. Precision $P(L)$. Precision [25] represents the proportion of the number of objects that are correctly predicted in the recommended list to the total number of recommended objects. The Precision of user $u_{i}$ is $d_{i}(L) / L$, and the average of all users' Precision is defined as follows:

$$
P(L)=\frac{1}{|M|} \sum_{u_{i} \in M} \frac{d_{i}(L)}{L},
$$

where $d_{i}(L)$ indicates that the number of objects predicted correctly in the recommendation list of user $u_{i}$ (i.e., the number of recommended objects appeared in the testing 


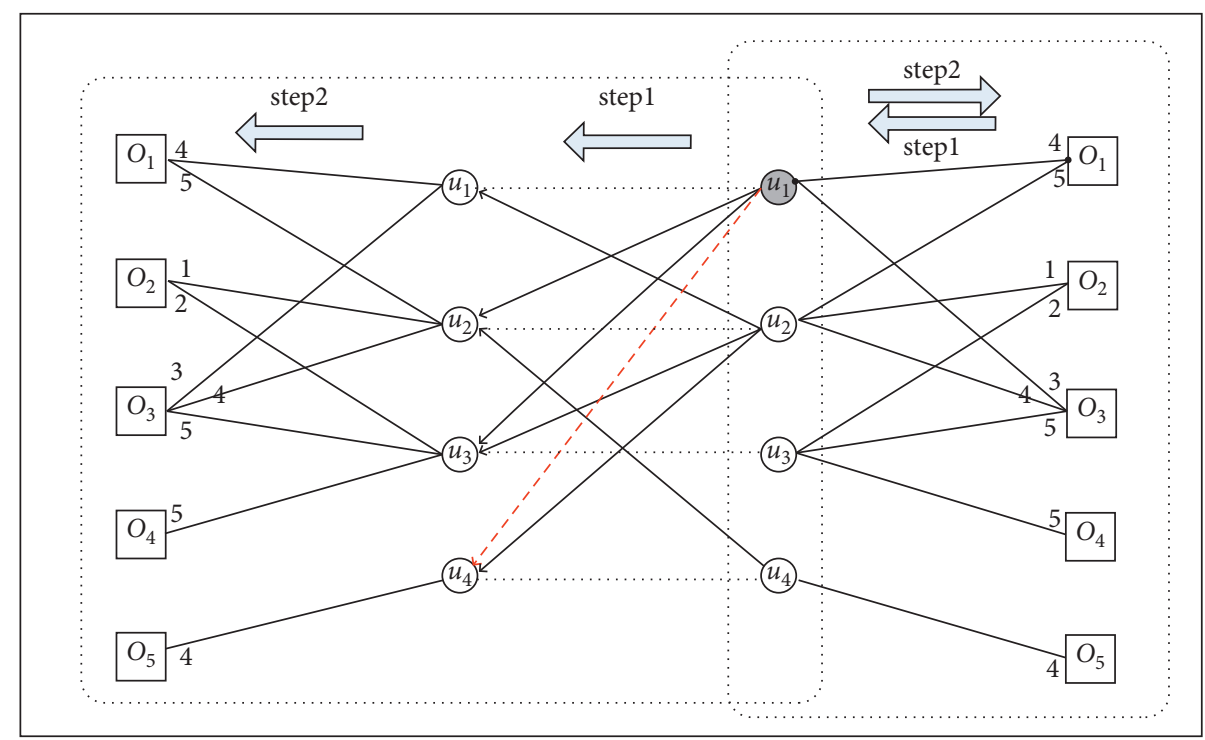

Figure 3: Dual wing mass diffusion model.

TABLE 1: Statistics of the datasets.

\begin{tabular}{lcc}
\hline & Epinions & Ciao \\
\hline \# of users & 22166 & 12375 \\
\# of objects & 296277 & 106797 \\
\# of categories & 27 & 28 \\
\# of rating & 922267 & 484086 \\
\# of links & 355813 & 237350 \\
Trust network density & 0.0014 & 0.0031 \\
\hline
\end{tabular}

set), and $M$ represents the user set. Here, the recommended objects that appeared in the testing set are regarded as correctly predicted objects.

3.2.2. Recall $R(L)$. Recall [26] represents the probability that the correctly predicted objects appear in the recommended list. Recall of user $u_{i}$ is the ratio of the number of correctly recommended objects $d_{i}(L)$ in the recommended list to the total number of candidate objects in the testing set, i.e., $d_{i}(L) /|T|$, and $T$ is the testing set. Recall of the system is the average of all users' Recall; the formula is as follows:

$$
R(L)=\frac{1}{|M|} \sum_{u_{i} \in M} \frac{d_{i}(L)}{|T|} .
$$

3.2.3. $F_{1}(L)$. As the length of the recommendation list increases, the Recall increases and Precision decreases. Therefore, $F_{1}(L)$ metric is introduced. $F_{1}(L)$ metric is the weighted average of Precision and Recall [27]. The calculation formula is as follows:

$$
F_{1}(L)=\frac{2 \times P(L) \times R(L)}{P(L)+R(L)} .
$$

3.2.4. Ranking Score RS. Ranking Score is a method of calculating the ranking of the correctly predicted object in the recommendation list [7]. The value of RS is calculated by the following:

$$
\mathrm{RS}=\frac{1}{|T|} \sum_{(i, \alpha) \in T} \frac{\operatorname{rank}_{i \alpha}}{N_{i}}
$$

where $\operatorname{rank}_{i \alpha}$ is the position of object $o_{\alpha}$ in recommendation list of user $u_{i} . N_{i}$ represents the number of objects that $u_{i}$ has not been collected in the testing set $T$. It can be seen from the formula that smaller RS suggested better accuracy.

3.2.5. Hamming Distance $H(L)$. Hamming distance [28] can measure the difference between the recommendation lists generated by the recommendation algorithm for different users. Suppose the length of recommendation list is $L$, and the number of overlapping objects in the recommended lists of users $u_{i}$ and $u_{j}$ is $Q_{i j}$, i.e., $Q_{i j}=\left|O_{i}^{L} \cap O_{j}^{L}\right|$, then Hamming distance of user $u_{i}$ and $u_{j}$ is $1-\left(Q_{i j} / L\right)$, The average value of Hamming distance is calculated by the following:

$$
H(L)=\frac{1}{|M|(|M|-1)} \sum_{u_{i}, u_{j} \in M, i \neq j} 1-\frac{Q_{i j}}{L} .
$$

Higher $H(L)$ value suggests stronger diversity of recommendations.

3.2.6. Intrasimilarity $I(L)$. Intrasimilarity [28] can be used to measure the similarity between objects in the recommendation list. Object similarity is calculated by $s_{\alpha \beta}=\left(1 / \sqrt{k_{\alpha} k_{\beta}}\right) \sum_{u_{i} \in M} a_{i \alpha} a_{i \beta}$.

Intrasimilarity of user $u_{i}$ is calculated by the following: 


$$
I_{i}(L)=\frac{1}{L(L-1)} \sum_{o_{\alpha}, o_{\beta} \in L_{i}, \alpha \neq \beta} s_{\alpha \beta},
$$

where $L_{i}$ represents the set of objects recommended to user $u_{i}$. The average value of intrasimilarity $I(L)$ is calculated by the following:

$$
I(L)=\frac{1}{|M|} \sum_{u_{i} \in M} I_{i} .
$$

Smaller intrasimilarity suggests better diversity.

3.2.7. Novelty $N(L)$. Novelty [20] measures the capacity of the recommendation algorithm to recommend unpopularity or unexpected objects. The less popular objects are more likely to make users feel novel and unexpected. Here, Novelty is defined by the average Popularity of recommended objects [28], written as follows:

$$
N(L)=\frac{1}{|M|} \sum_{u_{i} \in M}\left(\frac{1}{L} \sum_{o_{\alpha} \in L_{i}} k_{\alpha}\right),
$$

where $k_{\alpha}$ represents the number of users who collected objects $o_{\alpha}$. Lower $N(L)$ value suggests a higher novelty of recommendations.

\section{Experiments and Results}

4.1. Recommendation Performance. In order to verify the validity and superiority of DWMD proposed, our algorithm is compared with four benchmark methods on the Epinions and Cao data sets, which is the item-based collaborative filtering recommendation model (Item-based CF), mass diffusion (MD) [7], CosRA + T model, and trust-based mass diffusion model (TrustMD) [29]. In the Item-based CF model, it is recommended that the objects are similar to those collected by the target user, and the cosine similarity is used to calculate the object similarity. MD recommends the top $L$ objects for target users based on the bipartite network structure. CosRA + T introduces the trust relationship into the CosRA method, which combines the advantages of cosine similarity and the RA index. TrustMD allocates resources based on trust weight, which calculates based on the similar taste of objects.

Results of the algorithmic performance are summarized in Table 2. Here, the recommended list is set to 10, i.e., $L=10$. Compared with the benchmark methods, DWMD proposed in this paper achieved the best values for all metrics on the two data sets.

When evaluating the accuracy of the algorithm, $F_{1}(L)$ is employed to measure the comprehensive performance of the algorithm. DWMD has the highest F1 and smallest RS on both datasets, indicating that DWMD performs the best in recommendation accuracy among all the considered methods. Item-based CF performs the worst in recommendation accuracy as it gives the lowest values in both $P(L)$ and $R(L)$. The diversity metrics and novelty metrics in Table 2. $H(L)$ and $N(L)$ are employed to evaluate the diversity and novelty of the algorithm, respectively. It can be seen that the $H(L)$ of DWMD is $9.9 \%-12.3 \%$ higher than TrustMD, 6.9\%-12.7\% higher than CosRA + T, $18.2 \%-$ $22.6 \%$ higher than $\mathrm{MD}$, and $20.3 \%-14.8 \%$ higher than Itembased CF. Therefore, the diversity of DWMD is better than the benchmark methods. The $N(L)$ of DWMD is the best compared with the benchmark methods. By comparing the accuracy, diversity, and novelty of the recommendation algorithm, it can be seen that the accuracy, diversity, and novelty of DWMD are superior to the benchmark methods. This shows that introducing trust information to mass diffusion helps to improve the accuracy, diversity, and novelty.

4.2. The Impact of Recommendation List's Length. In order to further verify the effectiveness and superiority of the algorithm proposed in this paper, the performance of the algorithm is evaluated under different lengths of the recommendation list $L$. This part mainly examines the impact of the length $L$ on accuracy metrics $F_{1}(L)$, diversity metrics $H(L)$, and novelty metrics $N(L)$ by varying $L$ from 5 to 50 at step of 5 for datasets Epinions and Ciao. Figures 4 and 5 show the trend of three evaluation metrics of different algorithms with the recommended list length $L$ on the Epinions and Ciao datasets. Figure 4 presents the results regarding the accuracy metrics on both Epinions and Ciao datasets. The values of $F_{1}(L)$ first increase and then decrease as $L$ increases, where the values of $F_{1}(L)$ reach their maximum at $L=10$ on Epinions, while the values of $F_{1}(L)$ shows a gradual decrease on Ciao. Therefore, the values of $F_{1}(L)$ reach their maximum at $L=5$ on Ciao. We noticed that $F_{1}(L)$ of DWMD is optimal no matter how the length $L$ changes. That is, regardless of the length of the recommendation list, the accuracy of DWMD is better than other benchmark methods.

In Figure 4, the picture on the left is for Epinions and on the right is for Ciao. In the vertical axis, the accuracy metric is shown. On the horizontal axis, the length of the recommendation list $L$ increases from 5 to 50 .

Figure 5 presents the results regarding the diversity metric. The value of $H(L)$ slightly decreases as the increase of $L$ on both Epinions and Ciao datasets, indicating that the diversity decreases with the increase of the recommended list length. $H(L)$ of DWMD is always optimal no matter how the length $L$ changes, compared with the benchmark methods. The value of $N(L)$ declines rapidly with the increase of $L$, and the rate of decline slows down and becomes stable when $L$ is bigger than $10 . N(L)$ of DWMD is significantly lower than the other four benchmark methods. That is, the novelty of the DWMD method is much better than the benchmark methods. In summary, DWMD has the best diversity and novelty compared with the benchmark methods.

In Figure 5, two pictures on the left are for Epinions and two pictures on the right are for Ciao. In the vertical axis, the diversity metric and novelty metric are, respectively, shown. In the horizontal axis, the length of the recommendation list $L$ increases from 5 to 50 . 
TABLE 2: Values of the six-evaluation metrics after applying different recommendation algorithms on the two data sets. The length of the recommendation list is set as $L=10$. The results are averaged over 10 independent realizations. For each data set and each evaluation metric, the best result is emphasized by bold.

\begin{tabular}{|c|c|c|c|c|c|c|}
\hline Epinions & RS & $P(L)$ & $R(L)$ & $F_{1}(L)$ & $H(L)$ & $N(L)$ \\
\hline Item-based CF & 0.231 & 0.0260 & 0.0117 & 0.0081 & 0.609 & 243 \\
\hline $\mathrm{MD}$ & 0.187 & 0.0264 & 0.0159 & 0.0198 & 0.687 & 213 \\
\hline $\operatorname{CosRA}+\mathrm{T}$ & 0.179 & 0.0281 & 0.0192 & 0.0228 & 0.704 & 101 \\
\hline TrustMD & 0.176 & 0.0270 & 0.0259 & 0.0264 & 0.712 & 187 \\
\hline DWMD & 0.162 & 0.0289 & 0.0271 & 0.0280 & 0.831 & 87 \\
\hline Ciao & RS & $P(L)$ & $R(L)$ & $F_{1}(L)$ & $H(L)$ & $N(L)$ \\
\hline Item-based CF & 0.206 & 0.0211 & 0.0561 & 0.0307 & 0.593 & 271 \\
\hline MD & 0.144 & 0.0269 & 0.0623 & 0.0376 & 0.614 & 180 \\
\hline $\operatorname{CosRA}+\mathrm{T}$ & 0.123 & 0.0278 & 0.0620 & 0.0384 & 0.721 & 92 \\
\hline TrustMD & 0.126 & 0.0271 & 0.0650 & 0.0383 & 0.697 & 148 \\
\hline DWMD & 0.101 & 0.0299 & 0.0836 & 0.0440 & 0.790 & 70 \\
\hline
\end{tabular}
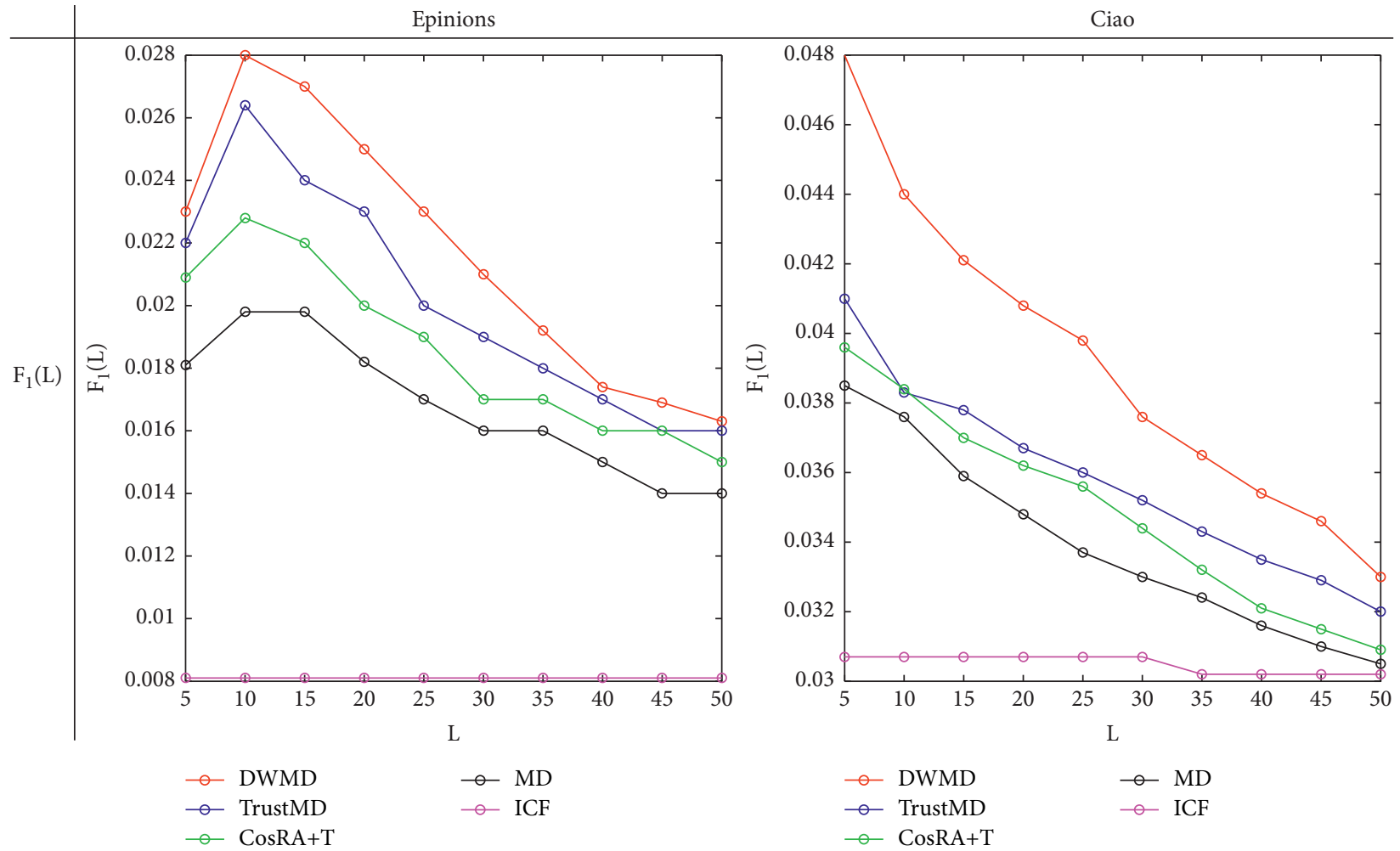

FIGURE 4: Results of the recommendation accuracy affected by the list's length.

4.3. The Impact of Tunable Parameter. In the proposed DWMD method, a tunable scaling parameter $\lambda$ is employed to adjust the proportion of resource from the user-userobject network with trust relations and from user-object network with object reputation. Figure 6 shows the influence of the parameter $\lambda$ on the performance of recommendations. $F_{1}(L), H(L)$, and $N(L)$ are, respectively, used as the evaluation metrics of accuracy, diversity, and novelty of recommendation performance. We vary $\lambda$ from 0 to 1 at the step of 0.1 , set the length of the recommendation list $L=10$, and evaluate it on the Epinions and Ciao datasets by employing the three accuracy metrics: $F_{1}(L), H(L)$, and $N(L)$. Figure 6 presents the corresponding results. In particular, when $\lambda=0$, the formula is a mass diffusion with object reputation algorithm. When $\lambda=1$, it is a trust network-based mass diffusion algorithm. It can be seen from Figure 4 that $F_{1}(L)$ and $H(L)$ both first increase and then decrease as $\lambda$ increases, while $N(L)$ first decreases and then increases as $\lambda$ increases on both datasets. It means that the introduction of additional trust information helps to improve the accuracy, diversity and novelty of recommendations, but the homogeneity of the trust relationship itself, when accumulated to a certain degree, will degrade the accuracy, diversity, and novelty. The accuracy, diversity, and novelty are not good when recommendations are made only through trust relationships or user-item collection 


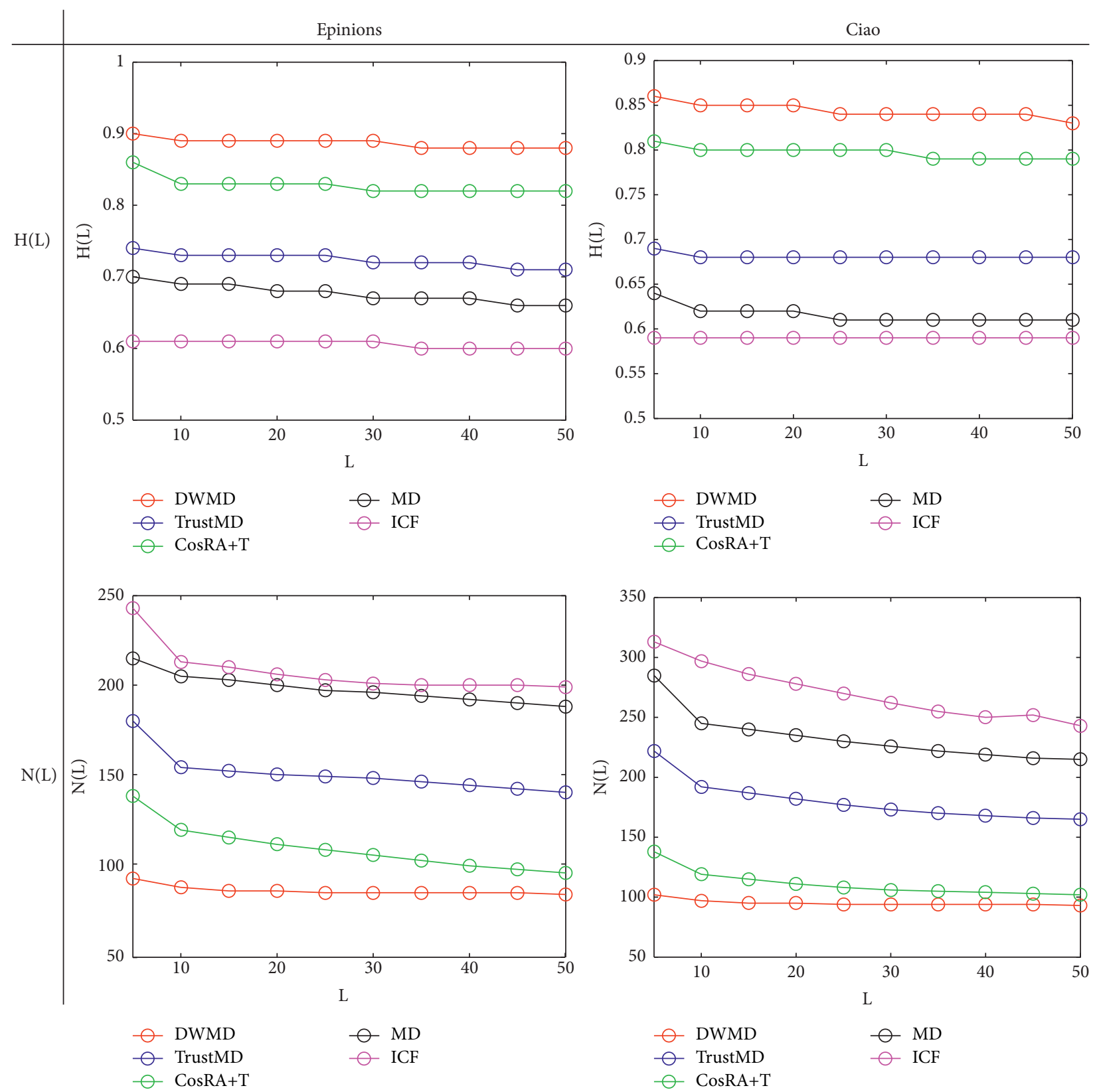

FIGURE 5: Results of the recommendation diversity and novelty affected by the list's length.

relationships with their evaluations. The combination of the two methods has the best recommendation performance. In the Epinions dataset, $F_{1}(L)$ and $H(L)$ get the highest values when $\lambda=0.8$, and the $N(L)$ is the best when $\lambda=0.4$; in the Ciao data set, $F_{1}(L)$ and $H(L)$ are the best at $\lambda=0.7$, and $N(L)$ is the best at $\lambda=0.3$. Therefore, the user can adjust the value of $\lambda$ according to the recommendation performance needed, so as to achieve the best recommendation performance.

In Figure 6, the three pictures above are for Epinions and the three pictures below are for Ciao. In the vertical axis, the accuracy metric, diversity metric, and novelty metric are, respectively, shown, including $F_{1}(L), H(L)$, and $N(L)$ (from the left to the right column). In the horizontal axis, the parameter $\lambda$ varies from 0 to 1 . The vertical dashed blue lines make the optimal value $\lambda$, where the accuracy metric, diversity metric, and novelty metric reach their maximum, respectively. The length of the recommendation list is set as $L=10$.

In order to study the effect of the recommended list length $L$ on the parameter $\lambda$, this part examines the accuracy $\left(F_{1}(L)\right)$ and the diversity $(H(L))$ according to the change of the recommended list lengths $L$, and takes the average value of the parameter $\lambda$ corresponding to the highest point of the index as the optimal value of the parameter $\lambda$. The average value of the parameter $\lambda$ corresponding to the highest point of the metric is taken as the optimal value of the parameter $\lambda$. In Figure $7, F_{1}(L)$ reaches the highest point at $\lambda=0.76$, 

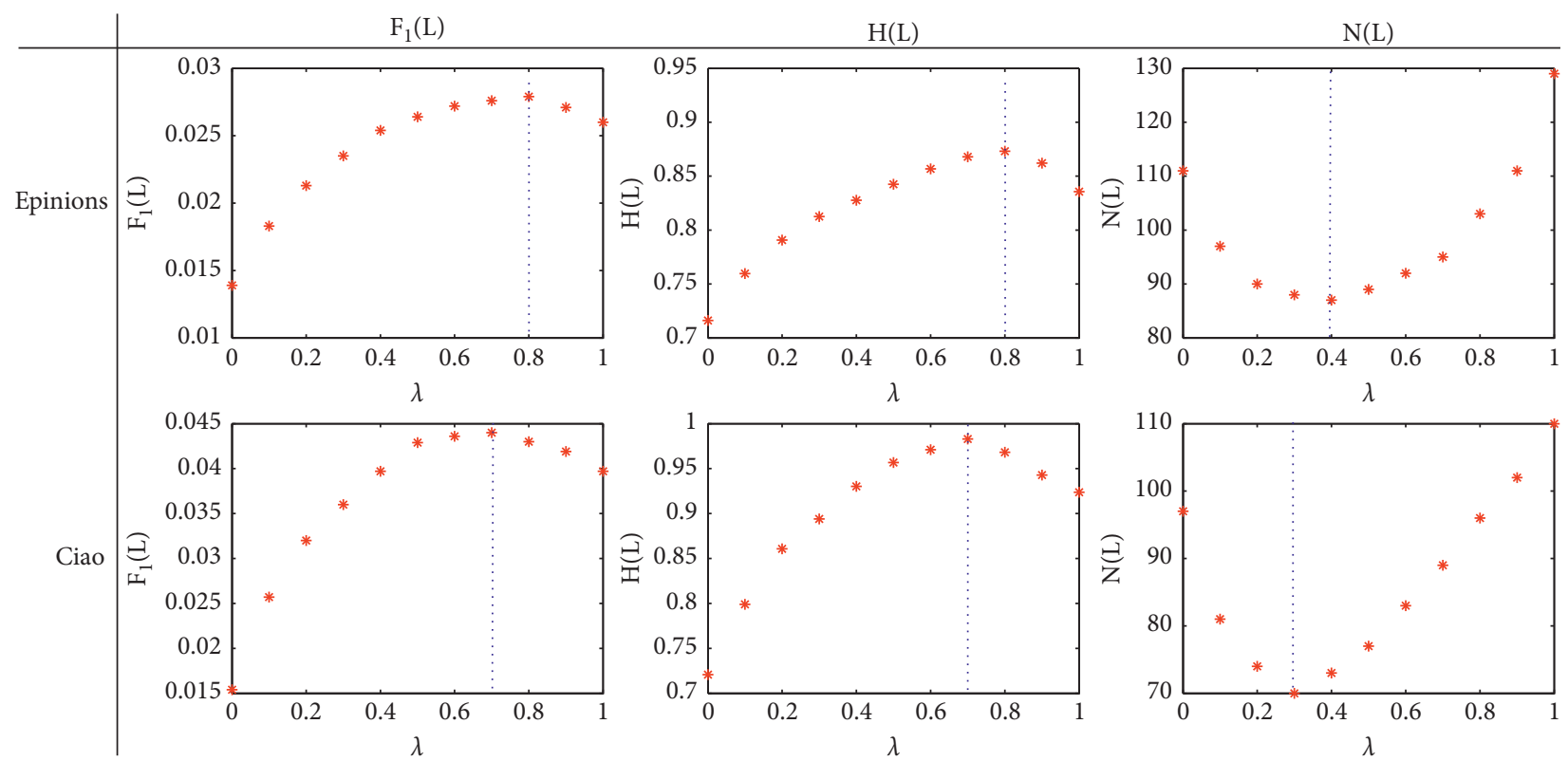

Figure 6: Results of the impact on parameter $\lambda$.
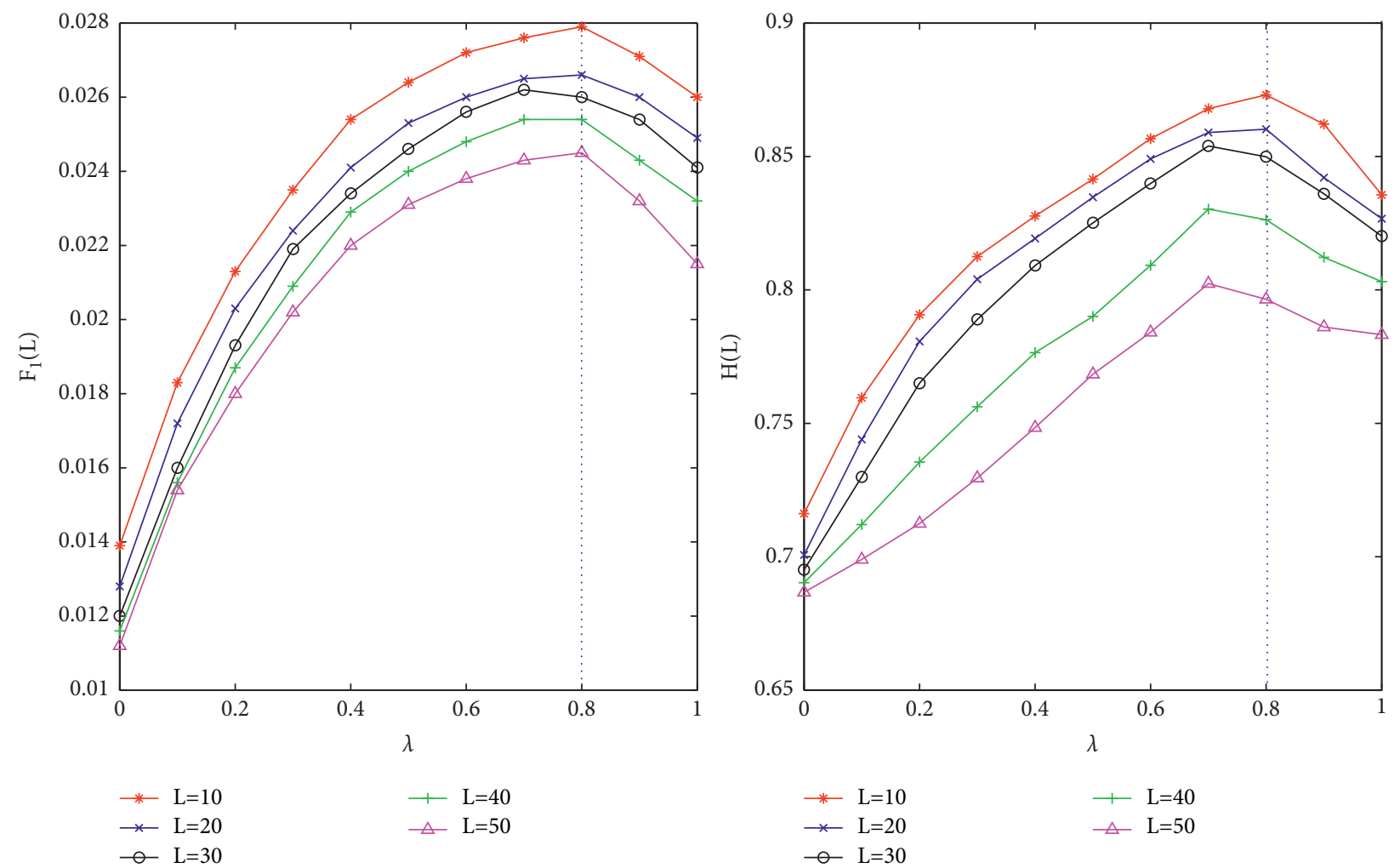

Figure 7: Results of parameter $\lambda$ affected by the list's length. The blue dotted line indicates the value of the parameter $\lambda$ when the accuracy and diversity are the best.

while $H(L)$ is the best when $\lambda=0.74$ on Epinions dataset. In this experiment, it was selected five lengths of the recommendation list. It can be observed that the length $L$ has little effect on parameter $\lambda$. The optimal value of parameter $\lambda$ indicates that the trust relationship can enhance the recommendation performance, but excessive reliance on the trust relationship will weaken the recommendation performance. 


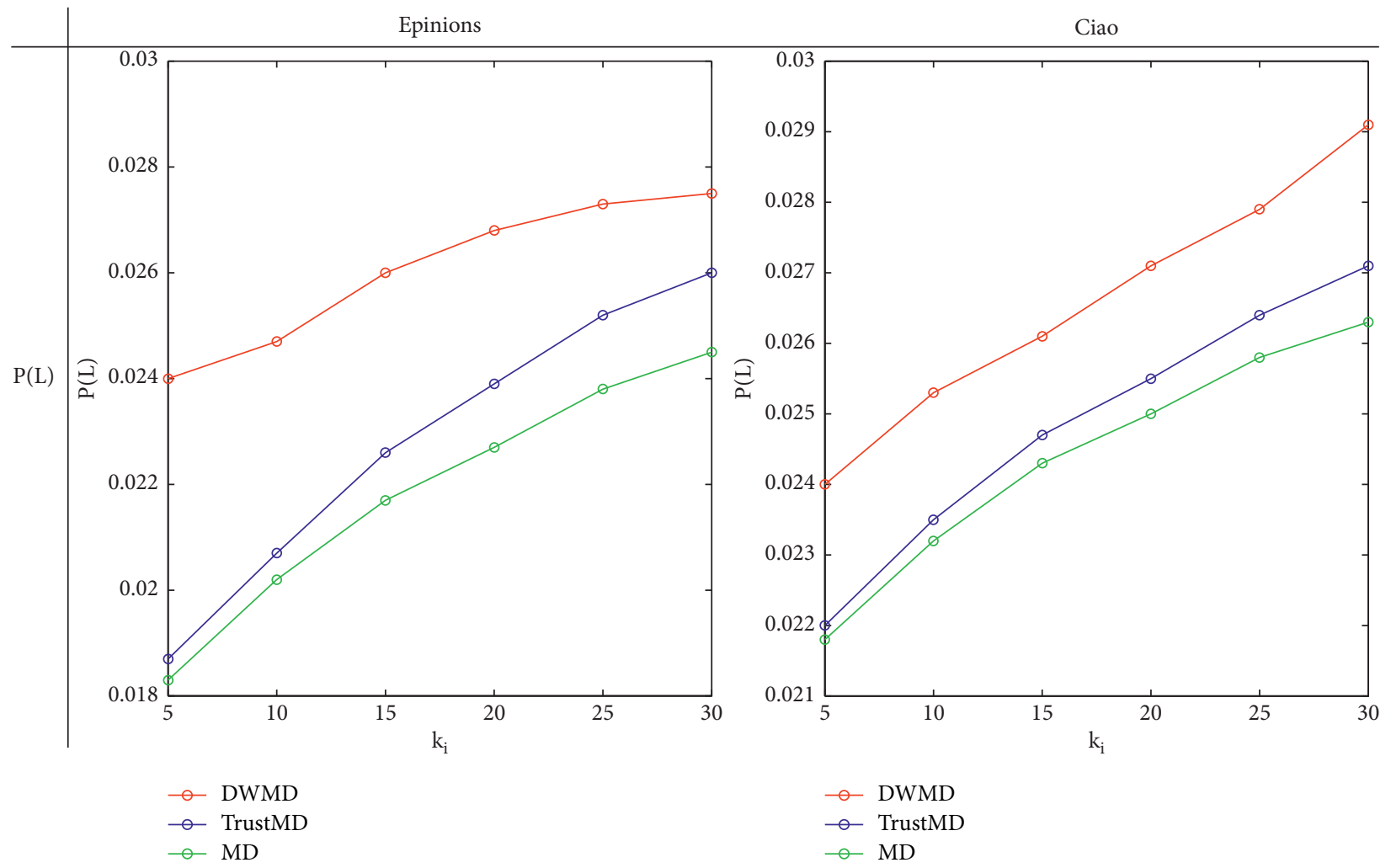

FIgURE 8: The impact of the number of objects collected on Accuracy $(P(L))$.

4.4. Recommendation Performance for Small-Degree Users. According to the statistics of the Epinions dataset, it is found that $65 \%$ of users collect the number of products between 11 and 30 , and the users who choose the number of products less than 100 accounted for $96 \%$. The same situation appeared in the Ciao dataset, where $72 \%$ of users collect objects less than 25 . The statistical data shows that the two datasets are reasonable as the research data for the recommendation performance analysis of small-degree users. When the small-degree user is used as the target user of the recommendation system, the Accuracy is not good due to less available information. Therefore, analyzing the recommendation performance of small-degree users can further reflect the pros and cons of the algorithm.

Here, users who collect less than 30 objects are regarded as small users, i.e., $k_{i} \leq 30$. Figure 8 shows how Accuracy $(P(L))$ changes according to the number of collected objects ( $\lambda$ is set to the optimal value, $5 \leq k_{i} \leq 30$ and the step is 5 ). It can be seen that no matter how $k_{i}$ changes within the range of value, the Accuracy of DWMD proposed in this paper is higher than that of MD and TrustMD, and the smaller the degree of the user, the greater the improvement of the Accuracy.

In order to further illustrate the advantages of DWMD to improve the accuracy of small-degree users' recommendation, DWMD is run on the complete network structure of the Epinions and Cao datasets, but only $P(L)$ of smalldegree users is calculated. From Table 3, it can be seen that TrustMD basically degenerates to MD on both Epinions and Cao datasets, indicating that TrustMD has basically no
TABle 3: $P(L)$ of different recommendation methods for Epinions and Ciao with $k_{i} \leq 30$.

\begin{tabular}{lccc}
\hline Dataset & MD & TrustMD & DWMD \\
\hline Epinions & 0.0219 & 0.0228 & 0.0261 \\
Cao & 0.0244 & 0.0249 & 0.0266 \\
\hline
\end{tabular}

advantage compared with MD when small-degree users as the target users. This is because TrustMD uses the collection relation to calculate the trust weight, which is the principle of resource allocation. TrustMD does not make full use of trust relationship information. However, the resource allocation weight of DWMD is calculated based on the structure of the trust relationship and has nothing to do with the object collection relationship. Therefore, the trust relationship can be fully utilized to improve recommendation accuracy when small-degree users as the target users. DWMD is $0.33 \%$ higher than TrustMD on accuracy, and $0.42 \%$ higher than MD. DWMD alleviates the generalized cold start problem by integrating social networks.

4.5. The Impact of Implicit Trust Relations. The trust network is composed of explicit trust relationships. Mining implicit trust relationships through explicit trust relationships can effectively reduce data sparsity. To explore the impact of implicit trust relationships, we compare DWMD with STMD and MD. DWMD recommends based on explicit and implicit trust relationships and fully considers the impact of user ratings. When $T_{i j}=0$, DWMD degenerates 
TABle 4: Performance comparison of different recommendation methods for Epinions and Ciao with $L=10$ and $L=5$, respectively.

\begin{tabular}{lcccc}
\hline Dataset & Epinions & $F_{1}(L)$ & $H(L)$ & $N(L)$ \\
\hline \multirow{3}{*}{ Epinions } & DWMD & $\mathbf{0 . 0 2 7 9}$ & $\mathbf{0 . 8 3 1}$ & $\mathbf{8 7}$ \\
& STMD & 0.0210 & 0.801 & 134 \\
& MD & 0.0198 & 0.687 & 213 \\
\hline \multirow{3}{*}{ Cao } & DWMD & $\mathbf{0 . 0 4 4 0}$ & $\mathbf{0 . 7 9 6}$ & $\mathbf{7 0}$ \\
& STMD & 0.0391 & 0.763 & 94 \\
& MD & 0.0376 & 0.614 & 180 \\
\hline
\end{tabular}

into STMD, i.e., the implicit trust relationship is not considered. The length of the recommendation list takes the best value in the experiments ( $L=10$ in the Epinions dataset, $L=$ 5 in the Cao dataset), and the results are shown in Table 4. It can be seen that the DWMD method performs better than STMD and MD on $F_{1}(L), H(L)$ and $N(L)$ for both Epinions and Ciao data sets, which shows that the implicit trust relationship helps to improve the accuracy, diversity, and novelty of recommendation. STMD is significantly better than the MD method, which indicates that explicit trust relationship and rating information can help improve the accuracy, diversity, and novelty of recommendation.

\section{Conclusion}

This paper studies the effect of introducing trust relationships and object reputation into the diffusion process to improve recommendation performance. The proposed DWMD method divides resources into two parts, which are, respectively, diffused on the user-user-object tripartite graph composed of trust relationships and user-object bipartite graph composed of object collection relations. The diffusion process on the two graphs allocates resources based on trust strength and object reputation, which weakens the popularity bias problem. DWMD uses the scaling parameter $\lambda$ to adjust the ratio of the two parts of the resource allocation, i.e., the ratio of the resource allocation to the user-object bipartite graph and the user-user-object tripartite graph. The existence of the optimal value of the parameter $\lambda$ indicates that the trust relationship can enhance the recommendation performance, but excessive dependence on trust will lead to the opposite result (the weakening of the recommendation performance). Results of extensive experiments based on the two real-world datasets, Epinions and Ciao, showed that the proposed DWMD method outperforms benchmark methods by giving a higher diversity and an encouraging novelty while ensuring a good accuracy in recommendations. Regarding the effects of the recommendation list's length on the performance evaluations under some parameter-dependent metrics (e.g., $F_{1}(L)$ and $H(L)$ ), we found that the optimal lengths of the recommendation list are nearly the same on the same dataset for different methods. We conducted a recommendation performance test especially for small-degree users, and found that the proposed DWMD method has a better performance than benchmark methods, effectively alleviating the generalized cold start problem of the recommendation algorithm for users who choose a small number of objects. This research provides a new way to improve recommendation performance, combining traditional user ratings and trust relationships with mass diffusion. This research puts forward a new perspective on using social networks for recommendation on heterogeneous networks, and verifies that the introduction of trust relationships will improve recommendation performance but overreliance on it will weaken performance.

\section{Data Availability}

The trust relationship data used to support the results of this study employed the data used in the study by Tang et al. You can download it from the URL: https://www.cse.msu.edu/ $\sim$ tangjili/trust.html.

\section{Conflicts of Interest}

The authors declare that they have no conflicts of interest.

\section{Acknowledgments}

This paper was supported by Science and Technology Planning Project of Yulin City (Project No. 2016-24-4) and Youth Project of Shaanxi Provincial Department of Science and Technology (Project No. 2021JQ-576).

\section{References}

[1] X. Su and T. M. Khoshgoftaar, "A survey of collaborative filtering techniques," Advances in artificial intelligence, vol. 2009, Article ID 421425, 2009.

[2] F. Tahmasebi, M. Meghdadi, S. Ahmadian, and K. Valiallahi, "A hybrid recommendation system based on profile expansion technique to alleviate cold start problem," Multimedia Tools and Applications, vol. 80, no. 2, pp. 2339-2354, 2021.

[3] S. Ahmadian, M. Afsharchi, and M. Meghdadi, "A novel approach based on multi-view reliability measures to alleviate data sparsity in recommender systems," Multimedia Tools and Applications, vol. 78, no. 13, pp. 17763-17798, 2019.

[4] S. Ahmadian, N. Joorabloo, M. Jalili et al., "A temporal clustering approach for social recommender systems," in Proceedings of the 2018 IEEE/ACM International Conference on Advances in Social Networks Analysis and Mining (ASONAM), pp. 1139-1144, IEEE, August 2018, Barcelona, Spain.

[5] S. Ahmadian, P. Moradi, and F. Akhlaghian, "An improved model of trust-aware recommender systems using reliability measurements," in Proceedings of the 2014 6th Conference on Information and Knowledge Technology (IKT), pp. 98-103, IEEE, Shahrood, Iran, May 2014.

[6] S. Ahmadian, M. Meghdadi, and M. Afsharchi, "Incorporating reliable virtual ratings into social recommendation systems," Applied Intelligence, vol. 48, no. 11, pp. 4448-4469, 2018.

[7] T. Zhou, J. Ren, M. Medo et al., "Bipartite network projection and personal recommendation," Physical Review, vol. 76, no. 4, Article ID 046115, 2007.

[8] Y.-C. Zhang, M. Blattner, and Y.-K. Yu, "Heat conduction process on community networks as a recommendation model," Physical Review Letters, vol. 99, no. 15, Article ID 154301, 2007.

[9] T. Zhou, Z. Kuscsik, J.-G. Liu, M. Medo, J. R. Wakeling, and Y.-C. Zhang, "Solving the apparent diversity-accuracy 
dilemma of recommender systems," Proceedings of the $\mathrm{Na}$ tional Academy of Sciences, vol. 107, no. 10, pp. 4511-4515, 2010.

[10] J. G. Liu, T. Zhou, and Q. Guo, "Information filtering via biased heat conduction," Physical Review E, vol. 84, no. 3, Article ID 037101, 2011.

[11] D.-C. Nie, Y.-H. An, Q. Dong, Y. Fu, and T. Zhou, "Information filtering via balanced diffusion on bipartite networks," Physica A: Statistical Mechanics and Its Applications, vol. 421, pp. 44-53, 2015.

[12] L. Lü and W. Liu, "Information filtering via preferential diffusion,” Physical Review E, vol. 83, no. 6, Article ID 066119 , 2011.

[13] X. Ren, L. Lü, R. Liu, and J. Zhang, "Avoiding congestion in recommender systems," New Journal of Physics, vol. 16, no. 6, Article ID 063057, 2014.

[14] Y.-C. Zhang, M. Medo, J. Ren, T. Zhou, T. Li, and F. Yang, "Recommendation model based on opinion diffusion," Europhysics Letters, vol. 80, no. 6, Article ID 68003, 2007.

[15] T. Zhou, R.-Q. Su, R.-R. Liu, L.-L. Jiang, B.-H. Wang, and Y.-C. Zhang, "Accurate and diverse recommendations via eliminating redundant correlations," New Journal of Physics, vol. 11, no. 12, Article ID 123008, 2009.

[16] D. Centola, "The spread of behavior in an online social network experiment," Science, vol. 329, no. 5996, pp. 1194-1197, 2010.

[17] J. Gao, T. Zhou, and Y. Hu, "Bootstrap percolation on spatial networks," Scientific Reports, vol. 5, no. 1, pp. 1-10, 2015.

[18] Q. Wang, J. Gao, T. Zhou, Z. Hu, and H. Tian, "Critical size of ego communication networks," EPL (Europhysics Letters), vol. 114, no. 5, Article ID 58004, 2016.

[19] H. Liao, G. Cimini, and M. Medo, "Measuring quality, reputation and trust in online communities," in Proceedings of the International Symposium on Methodologies for Intelligent Systems, pp. 405-414, Visakhapatnam, India, January 2012.

[20] X. Wang, Y. Liu, G. Zhang, F. Xiong, and J. Lu, "Diffusionbased recommendation with trust relations on tripartite graphs," Journal of Statistical Mechanics: Theory and Experiment, vol. 2017, no. 8, Article ID 083405, 2017.

[21] L. J. Chen, L. Gao, and H. Yang, "Information filtering ia trust relationships diffusion process," in Proceedings of the 2016 13th International Computer Conference on Wavelet Active Media Technology and Information Processing (ICCWAMTIP), pp. 76-81, IEEE, Chengdu, China, December 2016.

[22] L.-J. Chen and J. Gao, "A trust-based recommendation method using network diffusion processes," Physica A: Statistical Mechanics and Its Applications, vol. 506, pp. 679-691, 2018.

[23] L.-J. Chen, Z.-K. Zhang, J.-H. Liu, J. Gao, and T. Zhou, "A vertex similarity index for better personalized recommendation," Physica A: Statistical Mechanics and Its Applications, vol. 466, pp. 607-615, 2017.

[24] M. McPherson, L. Smith-Lovin, and J. M. Cook, "Birds of a feather: Homophily in social networks," Annual Review of Sociology, vol. 27, no. 1, pp. 415-444, 2001.

[25] J. Tang, H. Gao, H. Liu et al., "eTrust: Understanding trust evolution in an online world," in Proceedings of the 18th ACM SIGKDD international conference on Knowledge discovery and data mining, pp. 253-261, Beijing, China, August 2012.

[26] L. ü Linyuan, M. Medo, C. H. Yeung et al., "Recommender system,” Physics Reports, vol. 519, no. 1, pp. 1-49, 2012.

[27] L. Luo, H. Xie, Y. Rao, F. L. Wang et al., "Personalized recommendation by matrix co-factorization with tags and time information," Expert Systems with Applications, vol. 119, pp. 311-321, 2019.

[28] G. Chen, T. Gao, X. Zhu et al., "Personalized recommendation based on preferential bidirectional mass diffusion," Physica A: Statistical Mechanics and Its Applications, vol. 469, 2017.

[29] Y. Liu, L. Han, Z. Gou, and Y. Yang, "Personalized recommendation via trust-based diffusion," IEEE Access, vol. 7, pp. 94195-94204, 2019. 